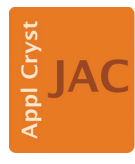

JOURNAL OF APPLIED CRYSTALLOGRAPHY

ISSN 1600-5767

Received 24 January 2017

Accepted 19 December 2017

Edited by S. Boutet, SLAC National Accelerator Laboratory, Menlo Park, USA

Keywords: X-ray diffraction; aerosols; nanocrystals.

\section{Femtosecond X-ray diffraction from an aerosolized beam of protein nanocrystals}

\author{
Salah Awel, ${ }^{\mathrm{a}, \mathrm{b}}$ Richard A. Kirian, ${ }^{\mathrm{c}}$ Max O. Wiedorn, ${ }^{\mathrm{a}, \mathrm{d}}$ Kenneth R. Beyerlein, ${ }^{\mathrm{a}}$ Nils \\ Roth, ${ }^{a}$ Daniel A. Horke, ${ }^{\mathrm{a}, \mathrm{b}}$ Dominik Oberthür, ${ }^{\mathrm{a}}$ Juraj Knoska, ${ }^{\mathrm{a}, \mathrm{d}}$ Valerio Mariani, ${ }^{\mathrm{a}}$ \\ Andrew Morgan, ${ }^{\text {a Luigi Adriano, }}{ }^{\text {e }}$ Alexandra Tolstikova, ${ }^{\text {a,d }}$ P. Lourdu Xavier, ${ }^{\text {a,f }}$ \\ Oleksandr Yefanov, ${ }^{a}$ Andrew Aquila, ${ }^{g}$ Anton Barty, ${ }^{a}$ Shatabdi Roy-Chowdhury, ${ }^{c}$ \\ Mark S. Hunter, ${ }^{\mathrm{g}}$ Daniel James, ${ }^{\mathrm{c}}$ Joseph S. Robinson, ${ }^{\mathrm{g}}$ Uwe Weierstall, ${ }^{\mathrm{c}}$ Andrei V. \\ Rode, ${ }^{\text {h }}$ Saša Bajt, ${ }^{\text {e }}$ Jochen Küpper ${ }^{\mathrm{a}, \mathrm{b}, \mathrm{d}}$ and Henry N. Chapman ${ }^{\mathrm{a}, \mathrm{b}, \mathrm{d} \text { * }}$
}

${ }^{a}$ Center for Free-Electron Laser Science, Deutsches Elektronen-Synchrotron DESY, Notkestrasse 85, 22607 Hamburg,
Germany, ${ }^{\mathbf{b}}$ The Hamburg Center for Ultrafast Imaging, Universität Hamburg, Luruper Chaussee 149, 22761 Hamburg,
Germany, ' Arizona State University, Tempe, Arizona, USA, ${ }^{\mathbf{d}}$ Department of Physics, Universität Hamburg, Luruper
Chaussee 149, 22761 Hamburg, Germany, ${ }^{\mathbf{e}}$ Photon Science, Deutsches Elektronen-Synchrotron DESY, Notkestrasse 85,
22607 Hamburg, Germany, ' Max-Planck Institute for the Structure and Dynamics of Matter, Luruper Chaussee 149,
22761 Hamburg, Germany, ${ }^{\mathbf{g}}$ Linac Coherent Light Source (LCLS), SLAC National Accelerator Laboratory, 2575 Sand Hill
Road, Menlo Park, CA 94025, USA, and hLaser Physics Centre, Research School of Physics and Engineering, Australian
National University, ACT 2601, Canberra, Australia. *Correspondence e-mail: henry.chapman@desy.de

High-resolution Bragg diffraction from aerosolized single granulovirus nanocrystals using an X-ray free-electron laser is demonstrated. The outer dimensions of the in-vacuum aerosol injector components are identical to conventional liquid-microjet nozzles used in serial diffraction experiments, which allows the injector to be utilized with standard mountings. As compared with liquid-jet injection, the X-ray scattering background is reduced by several orders of magnitude by the use of helium carrier gas rather than liquid. Such reduction is required for diffraction measurements of small macromolecular nanocrystals and single particles. High particle speeds are achieved, making the approach suitable for use at upcoming high-repetition-rate facilities.

\section{Introduction}

Serial femtosecond crystallography (SFX) allows the structural analysis of macromolecular crystals that may be too small or weakly scattering to study with synchrotron radiation sources. In order to record any measurable diffraction signal, such samples would require exposures far in excess of limits imposed by X-ray-induced radiation damage when using conventional sources. With typical pulse energies of about $1 \mathrm{~mJ}$, or $10^{12}$ photons, and durations of about $10 \mathrm{fs}$, pulses from $\mathrm{X}$-ray free-electron lasers (XFELs) overcome this limit by producing diffraction data before the onset of most damage processes (Neutze et al., 2000; Chapman et al., 2011; Boutet et al., 2012). Furthermore, XFELs enable novel time-resolved studies with femtosecond temporal resolution and ångström spatial resolution, all at physiological temperatures. A variety of prominent results from SFX measurements are summarized in recent reviews and special issues (Spence et al., 2012; Schlichting \& Miao, 2012; Barty et al., 2013; Patterson, 2014; Schlichting, 2015; Johansson et al., 2017).

The large increase in X-ray fluence afforded by the ability to outrun damage not only increases the diffraction signal from the sample but also increases the diffuse scattering from the medium transporting the crystal to the beam. Many SFX measurements were, therefore, performed on microcrystals 
that were large enough and ordered well enough to produce intense Bragg reflections that could be measured in the presence of the diffuse background. Such examples helped the rapid adoption of the technique. The use of such crystals, usually with volumes greater than $1 \mu \mathrm{m}^{3}$, enabled a broad range of sample delivery methods to be used depending on the nature of the experiment, such as liquid microjets (DePonte $e t$ al., 2008; Weierstall, 2014), viscous extrusion injectors (Weierstall et al., 2014) and solid substrates (Frank et al., 2014; Roedig et al., 2017). This flexibility is in stark contrast to efforts to record high-resolution coherent diffraction patterns from noncrystalline samples (Seibert et al., 2011; Küpper et al., 2014; Aquila et al., 2015; Yang et al., 2016). Without the amplification of the diffraction signal due to periodicity, objects such as molecules, viruses and other particles produce only weak scattering signals. Noncrystalline samples must, therefore, be delivered to the X-ray focus in a vacuum environment and in isolation from other potential scattering sources. This can be achieved, for example, through aerodynamic focusing of aerosolized particles (Bogan et al., 2008; Bogan, Boutet et al., 2010; Bogan, Starodub et al., 2010; Roth et al., 2017). In certain cases, background scattering from a dense surrounding medium is highly undesirable even for experiments on crystalline samples. For example, imaging techniques have been developed to utilize the faint continuous diffraction signal in regions between and at scattering angles beyond the intense Bragg reflections due to lattice disorder (Ayyer et al., 2016; Chapman et al., 2017) or lattice truncation (Spence et al., 2011; Kirian, Bean et al., 2015). The advantage and motivation for such approaches is that the continuous diffraction that can be accessed provides a direct route to solving the crystallographic phase problem without the need for prior knowledge or additional measurements.

Here, we demonstrate high-resolution X-ray diffraction from isolated protein nanocrystals delivered into the XFEL focus via a convergent-nozzle aerosol injector (CNAI) (Kirian, Awel et al., 2015). We show that the aerosol delivery produces extremely low background scattering signals compared with a conventional liquid jet. This aerosol injector has essentially the same size and form as the nozzles that are commonly used to produce liquid jets for SFX experiments (DePonte et al., 2008; Beyerlein et al., 2015; Oberthuer et al., 2017) and hence can be installed using standard liquid-jet mountings available at X-ray facilities. As shown in our previous work, CNAIs can produce aerosolized beams of submicrometre particles with a full-width at half maximum diameter $<5 \mu \mathrm{m}$ and particle speeds of the order of a few hundred metres per second, depending on particle size and operating conditions. This high velocity may be well suited to the megahertz repetition rates of upcoming XFEL sources.

\section{Experimental methods}

This proof-of-principle experiment was performed on natural Cydia pomonella granulovirus (GV) particles of approximately $200 \times 200 \times 370 \mathrm{~nm}$ in size that consist of a central virus body surrounded by a crystalline granulin protein shell.
They infect invertebrates such as the codling moth (Cydia pomonella) (Jehle et al., 2006). The GV particles used in this study were purified from a biopesticide solution (Certis Madex HP) using a method described elsewhere (Oberthuer et al., 2017) and suspended in water at a concentration of approximately $3 \times 10^{11}$ particles $\mathrm{ml}^{-1}$ prior to injection. The particle concentration was measured using a NanoSight (model LM14C) particle analysis system. The volume of the particle is about $0.015 \mu \mathrm{m}^{3}$, with about $2 / 3$ of that found as the volume of the crystalline shell (Gati et al., 2017), which corresponds to a diameter of approximately $300 \mathrm{~nm}$ for a sphere of equivalent volume. Despite the small size of these particles, previous SFX experiments recorded diffraction to $2.1 \AA$ resolution from such nanocrystals delivered to the X-ray beam in a gasfocused liquid jet (Gati et al., 2017).

Diffraction measurements were performed in the nanofocus chamber at the coherent X-ray imaging (CXI) (Liang et al., 2015) instrument at the Linac Coherent Light Source (LCLS). The experiment was carried out immediately after a successful liquid-jet experiment (Oberthuer et al., 2017) without disruption to the X-ray beam. During that earlier experiment the beam focus was optimized by adjusting the KirkpatrickBaez focusing mirrors via analysis of spot imprints on a gold foil. After optimization, the position of the beam was determined by placing an yttrium aluminum garnet (YAG) screen in the focal plane and observing optical fluorescence with a fixed in-line microscope with a resolution of a few micrometres. In our experiment, the aerosol beam was initially aligned relative to this reference and then scanned in position as described below.

The granulovirus suspension was aerosolized using a gas dynamic virtual nozzle (GDVN) (Beyerlein et al., 2015) mounted in a cylindrical nebulization chamber as depicted in Fig. 1(a). A GDVN uses gas-flow focusing to create a liquid jet with a diameter significantly smaller than the orifice of the nozzle, and which consequently breaks up to form a mist of droplets. The liquid was pressurized to flow from the nozzle at rates between 2.7 and $3.5 \mu \mathrm{min}^{-1}$, producing droplets of about $2 \mu \mathrm{m}$ diameter at a rate between $11 \times 10^{6}$ and $14 \times$ $10^{6} \mathrm{~s}^{-1}$, each containing on average 1.3 nanocrystals. This is equivalent to particle flow rates of $8.6 \times 10^{8}-1.1 \times$ $10^{9}$ particles $\min ^{-1}$.

The focusing gas was helium, which was set to a mass flow rate in the range of $10-60 \mathrm{mg} \mathrm{min}^{-1}$. The nebulization chamber had an inner diameter of approximately $40 \mathrm{~mm}$ and was $150 \mathrm{~mm}$ in length, giving a residence time in the chamber of several minutes, and a helium pressure that stabilized at a value between $100 \mathrm{mbar}$ and $1 \mathrm{bar}\left(1 \mathrm{bar}=10^{5} \mathrm{~Pa}\right)$. Under these conditions most of the solvent evaporated to produce nanocrystals suspended in a humid helium atmosphere (Kirian, Awel et al., 2015). Drops that contain more than one particle during the initial stage most likely form clusters of crystals (Cho et al., 2007; Daurer et al., 2017). The aerosol flowed through conductive silicone rubber tubing (Simolex, $6.3 \mathrm{~mm}$ inner diameter, $30 \mathrm{~cm}$ length), which was coupled to a standard 'nozzle rod' of the CXI beamline. This is a $1.2 \mathrm{~m}$ long stainless steel tube with a $6.3 \mathrm{~mm}$ inner diameter that is 
normally used to transfer liquid-jet injectors in and out of the main experimental chamber without breaking vacuum (Weierstall et al., 2012). The conductive tubing along the entire particle path acted as a Faraday cage to shield external electric fields from interacting with particles that might become charged through triboelectric effects in the GDVN. The aerosol finally exited the CNAI, which was mounted at the end of the CXI nozzle rod much like a typical liquid-jet nozzle. It consisted of a ceramic injection-molded tube of $1 \mathrm{~mm}$ outer diameter and $500 \mu \mathrm{m}$ inner diameter, a short converging section with a convergence angle of $15^{\circ}$, and a $100 \mu \mathrm{m}$ exit aperture (further details can be found in our previous work; Kirian, Awel et al., 2015).

During the diffraction experiment we monitored the crystal injection through direct optical imaging of scattered laser light from injected particles (Awel et al., 2016). A pulsed neodymium-doped yttrium lithium fluoride (Nd:YLF) laser (527 nm, $\sim 3 \mathrm{~mJ}$ per $\sim 150 \mathrm{~ns}$ pulse, $120 \mathrm{~Hz}$ ) was focused to a $\sim 0.8 \mathrm{~mm}$ spot within the aerosol stream, and scattered light was observed through the in-line microscope available at CXI [Questar long distance microscope, model QM-1 MK III, numerical aperture (NA) $=0.05$ at $750 \mathrm{~mm}$ objective distance]. Images were recorded using an OPAL-4000 CCD camera and stored at $30 \mathrm{~Hz}$. Fig. 1(c) shows a $3.7 \mathrm{~min}$ time-averaged optical image of particles exiting the injector. We determined that particles moved at speeds of approximately $300 \mathrm{~m} \mathrm{~s}^{-1}$ when they exited the injector, to arrive at the X-ray interaction point within a flight time of less than $1 \mu$ s. This particle speed was evaluated from the streak length of recorded particle images produced by laser illumination with a known pulse duration, conducted during laboratory characterization of the CNAI (see Fig. $2 a$ and $\$ 3$ ). The CNAI tip is seen to the left of Fig. 1(c), and the approximate X-ray focal point is indicated by the star. The particle stream could not be scattering from the tip saturated the imaging CCD. observed at points close to the CNAI tip because direct

3. Injector characterization and hit-fraction estimates In order to develop and characterize the operation of the CNAIs we conducted tests of both 15 and $30^{\circ}$ CNAIs in our laboratory. The setup differed from our previous work (Kirian, Awel et al., 2015) by the inclusion of a narrow particle transport tube intended to replicate the delivery system used at CXI. Aerosolized GV particles were transported from the nebulization chamber to the CNAI tip using stainless steel tubing of $4 \mathrm{~mm}$ inner diameter and $700 \mathrm{~mm}$ length. The GDVN was operated at flow rates of $2.7 \mu \mathrm{min}^{-1}$ and $28 \mathrm{mg} \mathrm{min}^{-1}$ for liquid sample and helium, respectively. A GV concentration of approximately $1.6 \times 10^{9}$ particles $\mathrm{ml}^{-1}$ was used (this was diluted by a factor of 200 from the solution that was used in the CXI experiment). This flow rate and sample concentration correspond to the generation of drops at a rate of approximately $1.1 \times 10^{7} \mathrm{~s}^{-1}$ and an entrance rate of aerosolized particles of $72 \times 10^{4} \mathrm{~s}^{-1}$.

The imaging setup used for visualizing particles was described in detail previously (Awel et al., 2016). Briefly, it was composed of an Nd:YLF laser (Spectra Physics Empower ICSHG-30, $527 \mathrm{~nm}$, approximate pulse duration $100 \mathrm{ns,}$ repetition rate $1 \mathrm{kHz}$, pulse energy $20 \mathrm{~mJ}$ ) to illuminate particles, a high-frame-rate CMOS camera (Photron SA4) and a $5 \times$ magnification, $0.14 \mathrm{NA}$ microscope objective to record images. The laser beam was collimated to a $2 \mathrm{~mm}$ spot, such that it illuminated particles across the entire field of view of the camera. The camera exposure time was set to $20 \mathrm{~ms}$, such that each frame contained 20 pulses of the $1 \mathrm{kHz} \mathrm{Nd:YLF}$ laser illumination. A single image of particles emerging from the CNAI is shown in Fig. 2(a). The images are streaked owing to the high speed of the particles, and the observed intensity profile of these streaks reflects the relatively fast rise and slow decay of the Nd:YLF laser pulse. Centroid positions of individual particle streaks contained in 23500 frames were used to produce the rate-corrected two-dimensional particle density map shown in Fig. 2(b). This rate-corrected density has units of particles per area per particle generation rate and is defined as

$$
D=N_{\mathrm{p}} /(A R),
$$

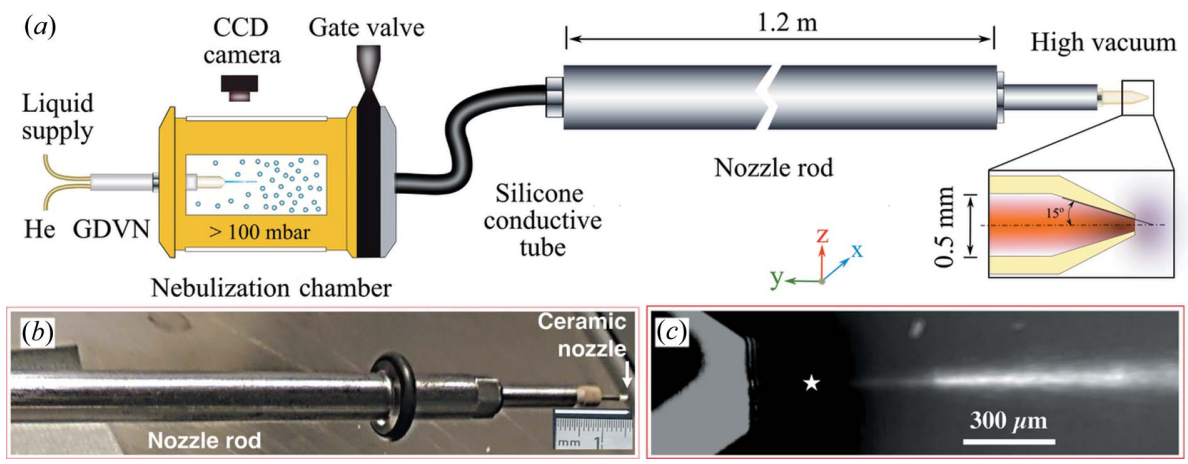

Figure 1

CNAI assembly and its operation during the CXI experiment. (a) Sketch of the basic aerosol generation and transportation setup. (b) The aerosol nozzle mounted on the nozzle rod. (c) Timeintegrated image of a laser-illuminated stream of GV particles exiting the CNAI, recorded using the inline microscope at the CXI instrument. This image was formed by averaging over $3.7 \mathrm{~min}$, with a running median background subtracted from each frame. The CNAI tip is seen in the left portion of the image, and the approximate X-ray focal point is indicated by the star. particles that fall within a spatial bin of area $A$, and $R$ is the rate at which particles entered the injector. Note that $N_{\mathrm{p}}$ represents the average particle counts at an instant in time and not a time integration over many exposures, which is appropriate because we intend to use the particle injector with femtosecond pulses. In our case, $N_{\mathrm{p}}$ was computed by summing the number of particles that fell within each spatial bin, and then dividing by the number of recorded images and the number of laser illumination pulses per image. where $N_{\mathrm{p}}$ is the average number of 
The measured rate-corrected density $D$ may be used to estimate the optimal hit fraction that could be achieved under idealized conditions in our X-ray measurements. If an entrance rate of $R_{\mathrm{X}}$ is used in the X-ray measurements, the two-dimensional particle number density is $D R_{\mathrm{X}}$. We define the effective cross-sectional area $\sigma$ such that the average number of particles intercepted by an X-ray pulse is $\sigma D R_{\mathrm{X}}$. Assuming Poisson statistics, the probability of intercepting just one particle in an X-ray pulse is

$$
H_{1}=\exp \left(-\sigma D R_{\mathrm{X}}\right) \sigma D R_{\mathrm{X}} \simeq \sigma D R_{\mathrm{X}},
$$

where the approximation holds to within $\sim 10 \%$ error as long as $\sigma D R_{\mathrm{X}}<0.1$. We define the $\mathrm{X}$-ray beam diameter as $d_{\mathrm{X}}$ and the particle beam diameter as $d_{\mathrm{p}}$ and estimate two limiting cases for the effective cross-sectional area. The first case, $\sigma^{+}=(\pi / 4)\left(d_{\mathrm{X}}+d_{\mathrm{p}}\right)^{2}$, describes the optimistic limit in which a particle at the periphery of the X-ray beam produces acceptable diffraction. The second case, $\sigma^{-}=(\pi / 4)\left(d_{\mathrm{X}}-d_{\mathrm{p}}\right)^{2}$, corresponds to the stronger assertion that an acceptable diffraction pattern requires that the entire X-ray beam width falls within the particle (if $d_{\mathrm{X}}<d_{\mathrm{p}}$ ) or that the entire particle falls within the X-ray beam width (if $d_{\mathrm{p}}<d_{\mathrm{X}}$ ). Finally, we arrive at two limiting hit-fraction estimates:

$$
H_{1}^{ \pm} \simeq \frac{\pi}{4} D\left(d_{\mathrm{X}} \pm d_{\mathrm{p}}\right)^{2} R_{\mathrm{X}}
$$

The maximum rate-corrected particle density recorded in the laboratory, i.e. at the focus of the particle beam shown in Fig. $2(b)$, was $D \simeq 2.2 \times 10^{-9} \mu \mathrm{m}^{-2}$ s. Assuming the approximate values $d_{\mathrm{X}} \simeq 150 \mathrm{~nm}, d_{\mathrm{p}} \simeq 300 \mathrm{~nm}$ and $R_{\mathrm{X}} \simeq 11 \times$ $10^{6} \mathrm{~s}^{-1}$ suggests that the maximum hit fraction to be expected in our XFEL diffraction measurements is in the range $H_{1}^{-} \simeq 0.04 \%$ to $H_{1}^{+} \simeq 0.4 \%$. This predicted hit fraction is much higher than the hit fraction we achieved during the CXI experiment, as discussed in the next section.

\section{X-ray diffraction analysis and discussion}

Diffraction measurements were conducted at a photon energy of $8 \mathrm{keV}$ and an estimated average pulse energy of $4.2 \mathrm{~mJ}$ prior to the $\sim 30-50 \%$ beamline transmission losses (Boutet, 2016). The CSPAD detector was located $127.9 \mathrm{~mm}$ downstream from the X-ray focus. We recorded detector data frames for every X-ray pulse, at a rate of $120 \mathrm{~Hz}$, for a cumulative total of $1.3 \mathrm{~h}$, which resulted in approximately 560000 data frames.

In all of our diffraction analysis we excluded all pixels from each detector frame that had abnormally high or low variances or mean values in 'dark' measurements made without X-rays, as well as a few patches of pixels for which there was obvious stray-light background. For every frame, the dark measurement was subtracted, and then a uniform common-mode electronic noise constant was subtracted from each detector panel. The common-mode offset was determined from unbonded detector pixels that are not sensitive to X-rays. The detector gain relating detector digital units to photon counts per pixel was obtained from a histogram of the pixel values, which yielded clear peaks corresponding to counts of zero, one and two photons. Most of this analysis was performed using the Python psana package provided by LCLS (Damiani et al., 2016).

Fig. 3 shows one quadrant of a recorded diffraction pattern from an aerosolized GV crystal, where the average detector dark frame and common-mode offsets have been subtracted. A total of 33 hits from $\mathrm{GV}$ were recorded, corresponding to a hit fraction of $\sim 0.006 \%$. Twenty-four patterns ( $73 \%$ of hits) were indexed using the CrystFEL software suite (White et al., 2012, 2016). Autoindexing failed on patterns that appeared to consist of multiple crystals clumped together. We expect the hit fraction for our aerosol injector to be significantly lower than a typical liquid jet (about 1-10\%) because of the $\sim 25$ fold higher particle speed of the aerosol beam and the approximately fourfold reduction in liquid flow rate. However, our recorded hit fraction was still lower than the range $0.04-0.4 \%$ that we estimated from our laboratory measurements.

For comparing the background obtained using the CNAI with that typically observed in liquid-jet experiments we examined data from a previous SFX experiment (Oberthuer et $a l ., 2017)$ in which the exact same GV sample was injected into the X-ray beam as a liquid suspension with a GDVN. All experimental parameters

Figure 2

Laboratory characterization of a beam of $\mathrm{GV}$ particles focused with the $15^{\circ}$ convergent aerosol nozzle using a strong-magnification imaging microscope. (a) A single exposure, showing streaked images of $\mathrm{GV}$ particles caused by the $100 \mathrm{~ns}$ laser illumination. The particles are moving from left to right and their streaked images have non-uniform intensity due to the relatively slow decay of the illumination laser pulses. $(b)$ The two-dimensional rate-corrected particle density determined from the centroids of individual particle images such as the one shown in $(a)$. (c) Gaussian fit to the particle density at the focal plane in $(b)$. 
were identical in both the CNAI and GDVN measurements except for the pulse energy, which was $4.6 \mathrm{~mJ}$ on average for the GDVN measurements.

The comparison of background scattering for the CNAI and GDVN approaches is presented in Fig. 4, which shows a plot of the normalized azimuthally averaged profiles of scatteredphoton counts (per pixel and per mJ of pulse energy), as a function of photon-wavevector transfer. The per-pixel standard deviations in the measurements are indicated by the gray regions in Fig. 4. The average profiles were divided by the average pulse energy to account for the slightly higher pulse energy in the case of the GDVN. The frames used in Fig. 4 were sampled uniformly from the final $\sim 5 \mathrm{~min}$ of data collection, when the conditions were closest to optimal, although little difference was noticed in other measurement segments. We excluded frames that fell below $1 \mathrm{~mJ}$ pulse energy. We additionally excluded frames that were visually corrupt as well as those for which the X-rays obviously missed the liquid jet, which corresponded to less than $10 \%$ of the frames. After removing these outliers, we confirmed that more than 10000 frames contributed to each of the two profiles.

As can be seen from the plots in Fig. 4, the liquid jet produces a background that is over 1000 times higher at a wavevector transfer of $q=2 \sin (\theta) / \lambda=0.32 \AA^{-1}$, corresponding to a resolution of $3.1 \AA$, where $\theta$ is the Bragg angle and $\lambda$ the wavelength. This coincides with the mean distance between oxygen atoms in water where diffuse scattering from water has its maximum. At low scattering angles the background from the liquid jet was about 200 times higher than for aerosol injection. The liquid jet for these measurements was operating at a flow rate of $20 \mu \mathrm{min}^{-1}$. Typical liquid flow rates needed to produce a stable jet range of $5-30 \mu \mathrm{min}^{-1}$,

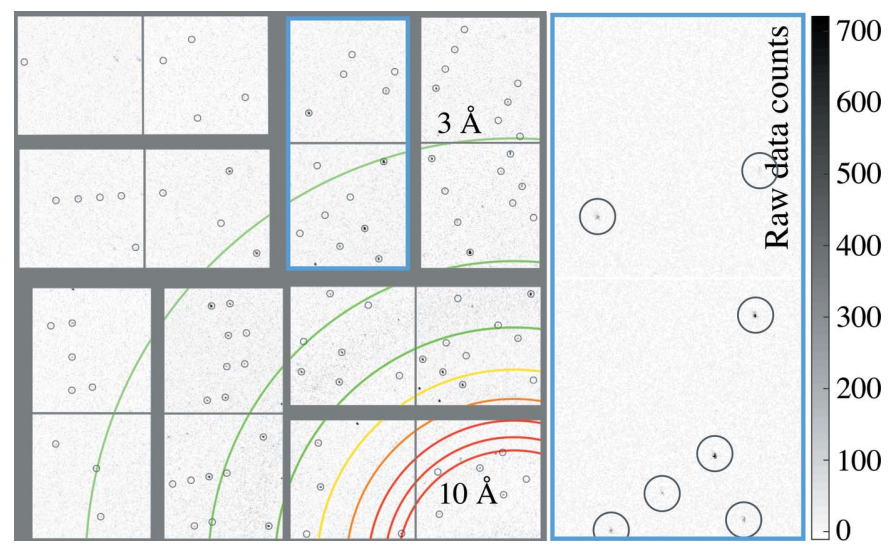

Figure 3

One detector quadrant of an indexed diffraction pattern obtained from aerosolized GV crystals. The colored rings indicate the resolution from 10 to $3 \AA$, in steps of $1 \AA$. The gray circles in the left-hand panel indicate the expected locations of Bragg peaks as determined by auto-indexing in the CrystFEL software suite (White et al., 2012, 2016). The right-hand panel shows an expanded view of an individual detector tile, marked by the blue rectangle on the left. Circles in this expanded-view panel indicate peaks that are easily recognizable by eye. Notably, the predicted peak locations indicated by CrystFEL do not perfectly agree with those that the human eye notices, but this is typical of first indexing results and could be improved through the CrystFEL post-processing routines. depending on the viscosity and surface tension of the liquid and the nozzle geometry. The volume of liquid that interacts with the X-ray beam scales roughly as the square root of the volumetric flow rate (Beyerlein et al., 2015), and thus the liquid-jet background is rather typical.

Because we use a convergent micro-focused particle beam, the hit fractions are highly sensitive to the relative positioning of the CNAI with respect to the X-ray beam. Our initial diagnostic for particle beam positioning was direct imaging of scattered light, which allowed for the rough positioning of the CNAI. From this initial position, it was necessary to perform a subsequent two-dimensional scan of the injector position in an effort to optimize the spatial overlap between particle beam focus and X-rays. Owing to the limitations of our $6 \mathrm{~h}$ measurement shift, we only performed one $200 \mu \mathrm{m}$ scan in the direction transverse to the particle beam and one $400 \mu \mathrm{m}$ scan along the particle beam direction. It is therefore highly unlikely that we located the ideal position that maximizes the hit fraction. However, we expect that the background scatter we observed is representative of the gas and water vapor exiting the injector because the gas expansion into vacuum is highly divergent. Direct imaging of the gas density leaving the CNAI (Horke et al., 2017) shows that the gas plume spans a volume hundreds of micrometres wide around the XFEL beam position.

Another possible culprit for our sub-optimal hit fraction is a sub-optimal aerosol transmission efficiency, which might be remedied by reducing the overall transportation tube length, by increasing the particle generation rate, by decreasing the particle speed, by increasing the volumetric flow rate of carrier gas or by the addition of aerodynamic lenses within the transport tube, which would maintain particles near the center

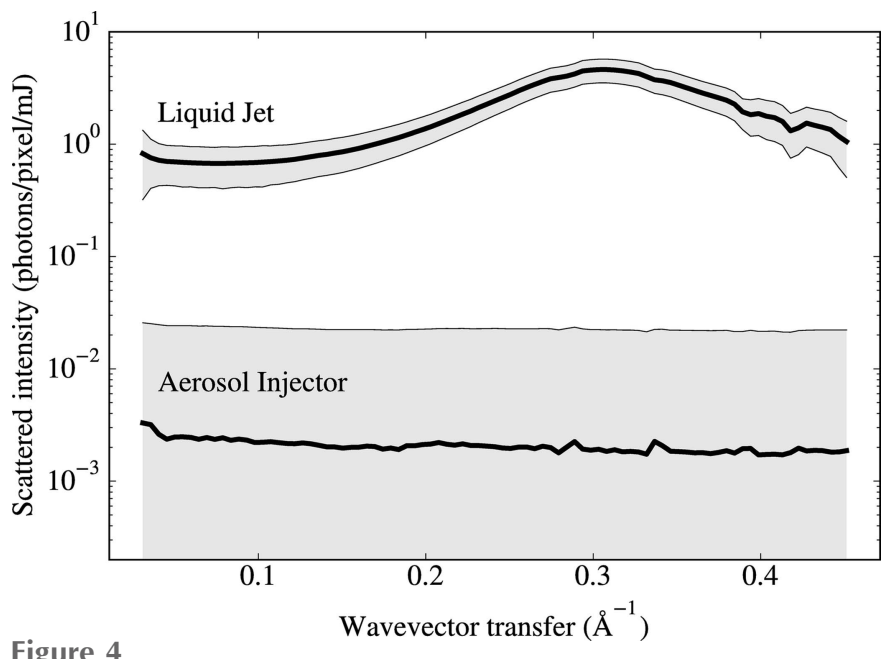

Figure 4

Average radial intensity profiles, on a logarithmic scale, for data measured using the GDVN (labeled 'Liquid Jet') and the CNAI (labeled 'Aerosol Injector') injectors. The average per-pixel standard deviations determined from more than 10000 frames are indicated by the vertical width of the gray regions. After averaging, the profiles and standard deviations were normalized by dividing by the average pulse energy, and then divided by the digital-to-photon conversion factor of 18.3. The horizontal axis corresponds to the wavevector transfer $q=2 \sin (\theta) / \lambda$, where $\theta$ is the Bragg angle and $\lambda$ is the wavelength. 
of the transport tube. Although aerosol injection hit fractions tend to be relatively low in comparison to liquid jets, recent work at the CXI instrument reported hit fractions of $0.83 \%$ for aerosolized $40 \mathrm{~nm}$ viruses delivered with an aerodynamic lens stack aerosol injector (Daurer et al., 2017).

Although it is convenient that our miniaturized CNAI is compatible with standard GDVN mounting hardware, the downside is that the small exit aperture, $100 \mu \mathrm{m}$ diameter in our case, is prone to clogging. We have successfully operated our CNAIs in the laboratory for many hours without interruption, but clogging typically occurs whenever the aerosolization liquid jet misbehaves and produces large droplets for a period of a few minutes. It is, therefore, essential to ensure the formation of small droplets and continuous flow of carrier gas. In the XFEL experiment reported here, there were a total of three clogged aerosol nozzles, each of which required 20-30 min to replace. The severity of this issue could be greatly reduced by filtering out large droplets with, for example, an in-line impactor (Maenhaut et al., 1996), and by using electrospray ionization to produce smaller initial droplet diameters (Yamashita \& Fenn, 1984; Chen et al., 1995; Bogan et al., 2008).

It must finally be noted that the GV crystals utilized here are notoriously robust and survive in nearly pure water. For crystals that dissolve, for instance, upon varying $\mathrm{pH}$, it may be feasible to avoid droplet evaporation by using a humidified carrier gas, by using electrospray nebulization or by simply placing the nebulization source close to the entrance of the aerosol nozzle to reduce the time of transport.

\section{Conclusions}

We have demonstrated X-ray diffraction from aerosolized sub-micrometre protein crystals with background levels drastically lower than in typical SFX experiments utilizing liquid jets. This may be important for coherent-diffractive-imaging experiments on weakly scattering targets such as isolated proteins, viruses or cells, as well as for the measurement of diffuse scattering or lattice-transform signals between crystalline Bragg reflections (Ayyer et al., 2016; Kirian, Bean et al., 2015). We showed that our injector is compatible with the existing hardware at LCLS, allowing quick changes from a liquid jet to an aerosol injection system in a single experiment. The relatively high $\left(\sim 300 \mathrm{~m} \mathrm{~s}^{-1}\right)$ particle speeds may be useful for avoiding damage due to X-ray-induced explosions when using new XFEL sources with pulse repetition rates up to 4.5 $\mathrm{MHz}$.

While the obtained $0.006 \%$ hit fraction at LCLS was much lower than in typical liquid-jet X-ray diffraction experiments, laboratory measurements suggest that this can be improved by orders of magnitude. On the basis of these laboratory measurements, we suspect that the low hit fractions observed in this study are a result of aerosol transport losses, clustering of particles, clogging of the aerosol nozzle due to an underperforming GDVN nebulizer, or misalignment between the $\mathrm{X}$-ray focus and particle beam focus. As we have noted, there are several possible routes to improve upon the injection strategy described here, as shown by other aerosol injection work performed at the same CXI instrument (Daurer et al., 2017).

Above all, the lower background achieved with the aerosol nozzle somewhat offsets the lower hit fraction, since the number of required measurements depends inversely on the square of the signal-to-noise ratio of intensities, or is directly proportional to the background counts.

This proof-of-principle experiment was performed on granulovirus occlusion bodies suspended in water. These protein crystals have naturally evolved to be robust against the change in the buffer conditions and dehydration caused by evaporation of the liquid layer on the crystals' surface. However, most protein crystals are not stable in pure water. When working with other types of crystals, the liquid buffer evaporation rate on the surface of the crystals must be controlled, for example by controlling the relative humidity at the crystals (Sanchez-Weatherby et al., 2009; Roedig et al., 2015).

\section{Acknowledgements}

We thank the LCLS staff for accommodating laser-light-scattering imaging in the CXI instrument during our experiments. Portions of this research were carried out at the LCLS at the SLAC National Accelerator Laboratory. This LCLS beamtime was part of the Protein Crystal Screening (PCS) program. LCLS is an Office of Science User Facility operated for the US Department of Energy, Office of Science, by Stanford University.

\section{Funding information}

Use of the Linac Coherent Light Source (LCLS), SLAC National Accelerator Laboratory, is supported by the US Department of Energy, Office of Science, Office of Basic Energy Sciences under contract No. DE-AC02-76SF00515. Parts of the sample delivery system used at LCLS for this research were funded by the NIH grant P41GM103393, formerly P41RR001209. In addition to DESY, this work has been supported by the excellence cluster 'The Hamburg Center for Ultrafast Imaging - Structure, Dynamics and Control of Matter at the Atomic Scale' of the Deutsche Forschungsgemeinschaft (CUI, DFG-EXC1074), the Gottfried Wilhelm Leibniz Program of the DFG, the European Research Council under the European Union's Seventh Framework Programme (FP7/2007-2013) through the Synergy Grant AXSIS (ERC-2013-SyG 609920) and the Consolidator Grant COMOTION (ERC-Küpper-614507), the Helmholtz Association 'Initiative and Networking Fund', and the Australian Research Council's Discovery Projects funding scheme (DP170100131). RAK acknowledges support from an NSF STC award (1231306).

\section{References}

Aquila, A. et al. (2015). Struct. Dyn. 2, 041701. 
Awel, S., Kirian, R. A., Eckerskorn, N., Wiedorn, M., Horke, D. A., Rode, A. V., Küpper, J. \& Chapman, H. N. (2016). Opt. Express, 24, 6507-6521.

Ayyer, K. et al. (2016). Nature, 530, 202-206.

Barty, A., Küpper, J. \& Chapman, H. N. (2013). Annu. Rev. Phys. Chem. 64, 415-435.

Beyerlein, K. R., Adriano, L., Heymann, M., Kirian, R., Knoška, J., Wilde, F., Chapman, H. N. \& Bajt, S. (2015). Rev. Sci. Instrum. 86, 125104.

Bogan, M. J., Benner, W. H. et al. (2008). Nano Lett. 8, 310-316.

Bogan, M. J., Boutet, S. et al. (2010). Aerosol Sci. Technol. 44(3), i-vi.

Bogan, M. J., Starodub, D., Hampton, C. Y. \& Sierra, R. (2010). J. Phys. B At. Mol. Opt. Phys. 43, 194013.

Boutet, S. (2016). Personal communication.

Boutet, S. et al. (2012). Science, 337, 362-364.

Chapman, H. N., Fromme, P. et al. (2011). Nature, 470, 73-77.

Chapman, H. N., Yefanov, O. M., Ayyer, K., White, T. A., Barty, A., Morgan, A., Mariani, V., Oberthuer, D. \& Pande, K. (2017). J. Appl. Cryst. 50, 1084-1103.

Chen, D.-R., Pui, D. Y. H. \& Kaufman, S. L. (1995). J. Aerosol Sci. 26, 963-977.

Cho, Y.-S., Yi, G.-R., Chung, Y. S., Park, S. B. \& Yang, S.-M. (2007). Langmuir, 23, 12079-12085.

Damiani, D., Dubrovin, M., Gaponenko, I., Kroeger, W., Lane, T. J., Mitra, A., O'Grady, C. P., Salnikov, A., Sanchez-Gonzalez, A., Schneider, D. \& Yoon, C. H. (2016). J. Appl. Cryst. 49, 672-679.

Daurer, B. J. et al. (2017). IUCrJ, 4, 251-262.

DePonte, D. P., Weierstall, U., Schmidt, K., Warner, J., Starodub, D., Spence, J. C. H. \& Doak, R. B. (2008). J. Phys. D Appl. Phys. 41, 195505.

Frank, M. et al. (2014). IUCrJ, 1, 95-100.

Gati, C. et al. (2017). Proc. Natl Acad. Sci. USA, 114, 2247-2252.

Horke, D. A., Roth, N., Worbs, L. \& Küpper, J. (2017). J. Appl. Phys. 121, 123106.

Jehle, J. A., Lange, M., Wang, H., Hu, Z., Wang, Y. \& Hauschild, R. (2006). Virology, 346, 180-193.

Johansson, L. C., Stauch, B., Ishchenko, A. \& Cherezov, V. (2017). Trends Biochem. Sci. 42, 749-762.

Kirian, R. A., Awel, S. et al. (2015). Struct. Dyn. 2, 041717.

Kirian, R. A., Bean, R. J., Beyerlein, K. R., Barthelmess, M., Yoon, C. H., Wang, F., Capotondi, F., Pedersoli, E., Barty, A. \& Chapman, H. N. (2015). Phys. Rev. X, 5, 011015.
Küpper, J. et al. (2014). Phys. Rev. Lett. 112, 083002.

Liang, M. et al. (2015). J. Synchrotron Rad. 22, 514-519.

Maenhaut, W., Hillamo, R., Mäkelä, T., Jaffrezo, J.-L., Bergin, M. H. \& Davidson, C. I. (1996). Nucl. Instrum. Methods Phys. Res. B, 109110, $482-487$.

Neutze, R., Wouts, R., van der Spoel, D., Weckert, E. \& Hajdu, J. (2000). Nature, 406, 752-757.

Oberthuer, D. et al. (2017). Sci. Rep. 7, 44628.

Patterson, B. D. (2014). Crystallogr. Rev. 20, 242-294.

Roedig, P., Ginn, H. M. et al. (2017). Nat. Methods, 14, 805-810.

Roedig, P., Vartiainen, I., Duman, R., Panneerselvam, S., Stübe, N., Lorbeer, O., Warmer, M., Sutton, G., Stuart, D. I., Weckert, E., David, C., Wagner, A. \& Meents, A. (2015). Sci. Rep. 5, 10451.

Roth, N., Awel, S., Horke, D. \& Küpper, J. (2017). arXiv:1712.01795 [physics.flu-Dyn].

Sanchez-Weatherby, J., Bowler, M. W., Huet, J., Gobbo, A., Felisaz, F., Lavault, B., Moya, R., Kadlec, J., Ravelli, R. B. G. \& Cipriani, F. (2009). Acta Cryst. D65, 1237-1246.

Schlichting, I. (2015). IUCrJ, 2, 246-255.

Schlichting, I. \& Miao, J. (2012). Curr. Opin. Struct. Biol. 22, 613626.

Seibert, M. M. et al. (2011). Nature, 470, 78-81.

Spence, J. C. H., Kirian, R. A., Wang, X., Weierstall, U., Schmidt, K. E., White, T. A., Barty, A., Chapman, H. N., Marchesini, S. \& Holton, J. (2011). Opt. Express, 19, 2866.

Spence, J. C. H., Weierstall, U. \& Chapman, H. N. (2012). Rep. Prog. Phys. 75, 102601.

Weierstall, U. (2014). Philos. Trans. R. Soc. London Ser. B, 369, 20130337.

Weierstall, U., James, D. et al. (2014). Nat. Commun. 5, 3309.

Weierstall, U., Spence, J. C. H. \& Doak, R. B. (2012). Rev. Sci. Instrum. 83, 035108.

White, T. A., Kirian, R. A., Martin, A. V., Aquila, A., Nass, K., Barty, A. \& Chapman, H. N. (2012). J. Appl. Cryst. 45, 335-341.

White, T. A., Mariani, V., Brehm, W., Yefanov, O., Barty, A., Beyerlein, K. R., Chervinskii, F., Galli, L., Gati, C., Nakane, T., Tolstikova, A., Yamashita, K., Yoon, C. H., Diederichs, K. \& Chapman, H. N. (2016). J. Appl. Cryst. 49, 680-689.

Yamashita, M. \& Fenn, J. B. (1984). J. Phys. Chem. 88, 44514459.

Yang, J. et al. (2016). Phys. Rev. Lett. 117, 153002. 